\title{
Propagation of intrinsic chirped sub-cycle and single-cycle pulses in a silica fiber
}

\author{
Xunming Cai ${ }^{\mathrm{a},{ }^{*}}, \quad$ Jingyun Zhao ${ }^{\mathrm{b}}, \quad$ Qiang Lin $^{\mathrm{c}}, \quad$ Jiaolian Luo ${ }^{\mathrm{a}}$, Yang Yang ${ }^{\mathrm{a}}$
}

${ }^{a}$ Special and Key Laboratory of Guizhou Provincial Higher Education for Green Energy-Saving Materials. Guizhou Minzu University, Guiyang 550025, China

${ }^{\mathrm{b}}$ School of Science, Zhejiang Sci.-Tech. University, Hangzhou 310018, China

${ }^{c}$ Department of Applied Physics, College of Science, Zhejiang University of Technology, Hangzhou 310023, China

\begin{abstract}
The propagation of Gaussian sub-cycle and single-cycle pulses in a nonlinear media is studied using the analytical expression of pulses. The analytical expression is a modified version of the vector potential definition model of sub-cycle pulse. The intrinsic characteristics of sub-cycle and single-cycle pulses, such as the intrinsic chirp and the self-induced blue-shift of the central frequency of spectrum are found to have an important effect on the propagation of pulses in the nonlinear media. The initial 0.28 -cycle pulse evolves into a primary multi-cycle pulse and a single-cycle precursor pulse during the propagation. The single-cycle precursor pulse is formed by the carrier frequency modulation on the leading side of pulse. During the propagation of the sub-cycle pulse, the self-steepening effect and the amplitude of the precursor pulse are more significant due to the shorter pulse duration. The reason can be attributed to the intrinsic characteristics of sub-cycle pulse.
\end{abstract}

Key words: Sub-cycle pulse; Intrinsic chirp; Self-steepening effect; Carrier frequency modulation

\section{Introduction}

The development of ultrafast optical technology enables the generation of ultrafast pulses containing only a few cycles or even less than one optical cycle [1,2]. The few-cycle, single-cycle and sub-cycle pulses start a new era for ultrafast science. A lot of work has been performed on the theoretical description and experimental application of the single-cycle and sub-cycle pulses and their interaction with matter as well [3-7].

The propagation of ultrashort pulse in nonlinear media is an important and interesting problem and much work has been done on the topic. Generally, the Generalized nonlinear Schrödinger(GNS) equation is applied to research the propagation of ultrashort pulse in nonlinear media. The beam-propagation method is an important tool for solving GNS equation under the slowly varying envelope(SVE) approximation. However, the SVE approximation is invalid if the pulse duration approaches the optical cycle regime. It is necessary to use the finite-difference time-domain (FDTD) method to solve the Maxwell's equations beyond the SVE approximation [8-12]. In Ref [9], the results of this extended FDTD method are compared with experimental results for the nonlinear propagation of a 12-fs chirped laser pulse in a

\footnotetext{
* Corresponding author

E-mail address:caixm1997@aliyun.com
}

(C) 2016. This manuscript version is made available under the Elsevier user license

http://www.elsevier.com/open-access/userlicense/1.0/ 
silica fiber. The FDTD calculation of Maxwell's equations has been extended to include all exact Sellmeier-fitting values. The general vector auxiliary differential equation (GVADE) FDTD method is developed in Ref [13], the GVADE FDTD method is a high effect algorithm and can be used to model spatial soliton propagation with two orthogonal electric field components in fused silica. A new method evolved from split-step fourier method for studying the propagation of optical pulses through nonlinear dispersive media is developed in [14-15]. Recently, the general overviews of the experimental and theoretical research of few-cycle pulse in nonlinear media are reported in the references [16-17].

When the pulse duration approaches the optical cycle, the direct current (DC) component in carrier-envelope (CE) model with an envelope multiplying with a carrier wave is found to cause nonphysical results [18]. Recently, the analytical expressions of single-cycle and sub-cycle pulses which are the exact solutions of Maxwell's equations have been found $[19,20]$. Some new properties of sub-cycle pulse including the intrinsic chirp and self-induced blueshift of the center frequency are discovered. In this paper, the propagation of Guassian sub-cycle pulse is studied in fused silica. The same chromatic dispersion, nonlinear Kerr polarization, and nonlinear Raman polarization are used as in Refrence [9], which model realistic physical effects in a silica fiber. It is found that the initial 0.28-cycle pulse evolves into a primary multi-cycle pulse and a single-cycle precursor pulse after the propagation time of 5.344 picoseconds. The intrinsic characteristics of sub-cycle pulse are found to have an important effect on the amplitude of the precursor pulse. The self-steepening effect is very clear for the sub-cycle pulse.

\section{Description of gaussian sub-cycle pulse}

As mentioned before, the carrier-envelope (CE) model is no longer applicable for the sub-cycle and single-cycle pulses, the direct-current (DC) component existing in the carrier-envelope model is found to cause nonphysical effects. So, the pulse expressions derived from the vector potential definition have been developed to cancel out the DC part in the single-cycle and sub-cycle fields. The vector potential of pulse is given as

$$
A(t)=\operatorname{Re}\left[-\frac{c}{i \omega_{0}} E_{0} f(t) \exp \left(i \omega_{0} t+i \phi_{0}\right)\right],
$$

where $\omega_{0}=2 \pi / T_{0}$ is the carrier frequency, $T_{0}$ is the period, $\phi_{0}$ represents the absolute phase or CEP, $E_{0}$ is the amplitude and $f(t)$ is a real Guassian-like envelope function. The electric field is derived as the first derivative of the vector potential. The envelope function satisfies

$$
\lim _{t \rightarrow+\infty} f(t)=0
$$

The E field is guaranteed to have zero DC component.

For a propagable pulse, the removing of DC component is only the first requirement. $f(t)$ is only physically meaningful when the spectrum content remains invariant under the change of its carrier-envelop-phase (CEP) [18-19]. As the Fourier spectrum $E(\omega)$ isn't dependent on the phase, the weighted center frequency $\omega_{c}=\int_{0}^{\infty} \omega|E(\omega)|^{2} d \omega / \int_{0}^{\infty}|E(\omega)|^{2} d \omega$ should remain unchanged when CEP changes. As pointed out in Refs [21], the vector potential model remains high accuracy for pulse durations down to three quarter cycles $\left(\tau_{p} \approx 0.75 T_{0}\right)$, but 
fails for even shorter ones. The pulse width $\tau_{p}$ is defined as the full-width at half-maximum (FWHM) of envelope functions $f(t)$. The reason why the vector potential definition works in the long pulse limit but fails when the pulse is short enough could be explained by the Bedrosian Theory.

To cover the whole range of pulse width, we can modify the vector potential definition by assuming $f(t)$ to be an analytical envelope $\tilde{f}(t)=u(t)+i \hat{u}(t)$, where real function $u(t)$ and $\hat{u}(t)$ is a Hilbert transform pair. The analytical Lorentzian envelope is given in Ref [21]. So the derived E field expression becomes an analytical function and also the DC part is removed. The analytical Lorentzian envelope can fulfill the condition that its spectrum content remains invariant under the change of its CEP. But the Lorentzian envelope decays slowly in time domain and isn't commonly used in practical application. In the paper, the analytical Guassian envelop is given. The Fourier spectrum $E(\omega)$ remains unchanged when the CEP changes for the pulse durations down to three-tenths cycle $\left(\tau_{p} \approx 0.3 T_{0}\right)$.

The analytical Guassian envelope is expressed as

$$
\tilde{f}(t)=u(t)+i \hat{u}(t)=e^{-\left(\frac{t}{T}\right)^{2}}\left(1+\frac{2 i}{\sqrt{\pi} T} \int_{0}^{t} e^{\left(\frac{t^{\prime}}{T}\right)^{2}} d t^{\prime}\right),
$$

where $T$ is the pulse width parameter. In order to avoid the broadening of envelop in time domain, a normalized factor is introduced to the function (3). So the modified Guassian envelope function can be written as

$$
\tilde{f}^{\prime}(t)=e^{-\left(\frac{t}{T}\right)^{2}}\left(1+\frac{2 i}{\sqrt{\pi} T} \int_{0}^{t} e^{\left(\frac{t^{\prime}}{T}\right)^{2}} d t^{\prime}\right) / \sqrt{1+\left(\frac{2}{\sqrt{\pi} T} \int_{0}^{t} e^{\left(\frac{t^{\prime}}{T}\right)^{2}} d t^{\prime}\right)^{2}}=e^{-\left(\frac{t}{T}\right)^{2}} s_{1} / s_{2},
$$

where $s_{1}=1+\frac{2 i}{\sqrt{\pi} T} \int_{0}^{t} e^{\left(\frac{i}{T}\right)^{2}} d t^{\prime}$ and $s_{2}=\sqrt{1+\left(\frac{2}{\sqrt{\pi} T} \int_{0}^{t} e^{\left(\frac{t^{\prime}}{T}\right)^{2}} d t^{\prime}\right)^{2}} . \quad$ By taking a first time derivative, the E field could be derived and normalized to be

$$
E(t)=\operatorname{Re}\left[E_{0}\left|A_{d}(0)\right|^{-1} A_{d}(t) \tilde{f^{\prime}}(t) \exp \left(i \omega_{0} t+i \phi_{0}\right)\right],
$$

The expression is similar to the CE expression except the self-modulation factor $\left|A_{d}(0)\right|^{-1} A_{d}(t)$. The function

$$
A_{d}(t)=1-i \frac{\tilde{f^{\prime}}}{\omega_{0} \tilde{f}}
$$

is the self-modulation function, where $\tilde{f}^{\prime}$ is the first time derivative of $\tilde{f}^{\prime}$. Under the slowly varying envelope approximation, the change of the envelope is much slower than the rapid oscillating carrier wave, so the expression reduces to the standard CE expression with $\left|A_{d}(0)\right|^{-1} A_{d}(t)=1$. The specific expression of Guassian envelope function is given as 


$$
E(t)=\frac{E_{0}}{1+2 /\left(\omega_{0} T \sqrt{\pi}\right)} \operatorname{Re}\left(A_{d}(t) \tilde{f^{\prime}}(t) e^{i \omega_{0} t+i \phi_{0}}\right),
$$

where

$$
A_{d}(t)=1+i\left(2 / T^{2} t e^{-\left(\frac{t}{T}\right)^{2}} s_{1} / s_{2}+(2 /(\sqrt{\pi} T))^{2} s_{1} s_{2}^{-3} \int_{0}^{t} e^{\left(\frac{t}{T}\right)^{2}} d t^{\prime}-2 i /\left(T s_{2} \sqrt{\pi}\right)\right) /\left(\omega_{0} \tilde{f^{\prime}}(t)\right) .
$$

The total phase of this pulse is expressed as follows:

$$
\phi(t)=\omega_{0} t+\phi_{0}+\arg \left(\tilde{f}^{\prime}(t)\right)+\arg \left(A_{d}(t)\right) .
$$

As shown in Refs [19], the corresponding instantaneous frequency $\omega_{e}(t)=d \phi(t) / d t$ satisfies

$$
\omega_{e}(0) \geq \omega_{e}(t)>\omega_{0} .
$$

Hence, the corresponding instantaneous frequency is highest at the center of the pulse and blueshifted with respect to the carrier-wave frequency $\omega_{0}$ throughout the entire pulse. Fig.1(a) shows the time waveform of three-tenths cycle Gaussian pulse. The dependence of frequency spectrum on the CEP is shown in Fig.1(b). The center frequency of pulse significantly exceeds the carrier frequency, which is so-called the self-induced blueshift. From Fig.1(c), one can see that the instantaneous frequency is symmetrical within the pulse and lowest on both the leading side and the trailing side of pulse. In Fig.1, the FWHM of pulse is $0.28 T_{0} \quad\left(\tau_{p}=2 \sqrt{\ln 2} T\right)$. The carrier frequency of pulse is $\omega_{0}=1.655 \times 10^{15} \mathrm{~Hz}$.
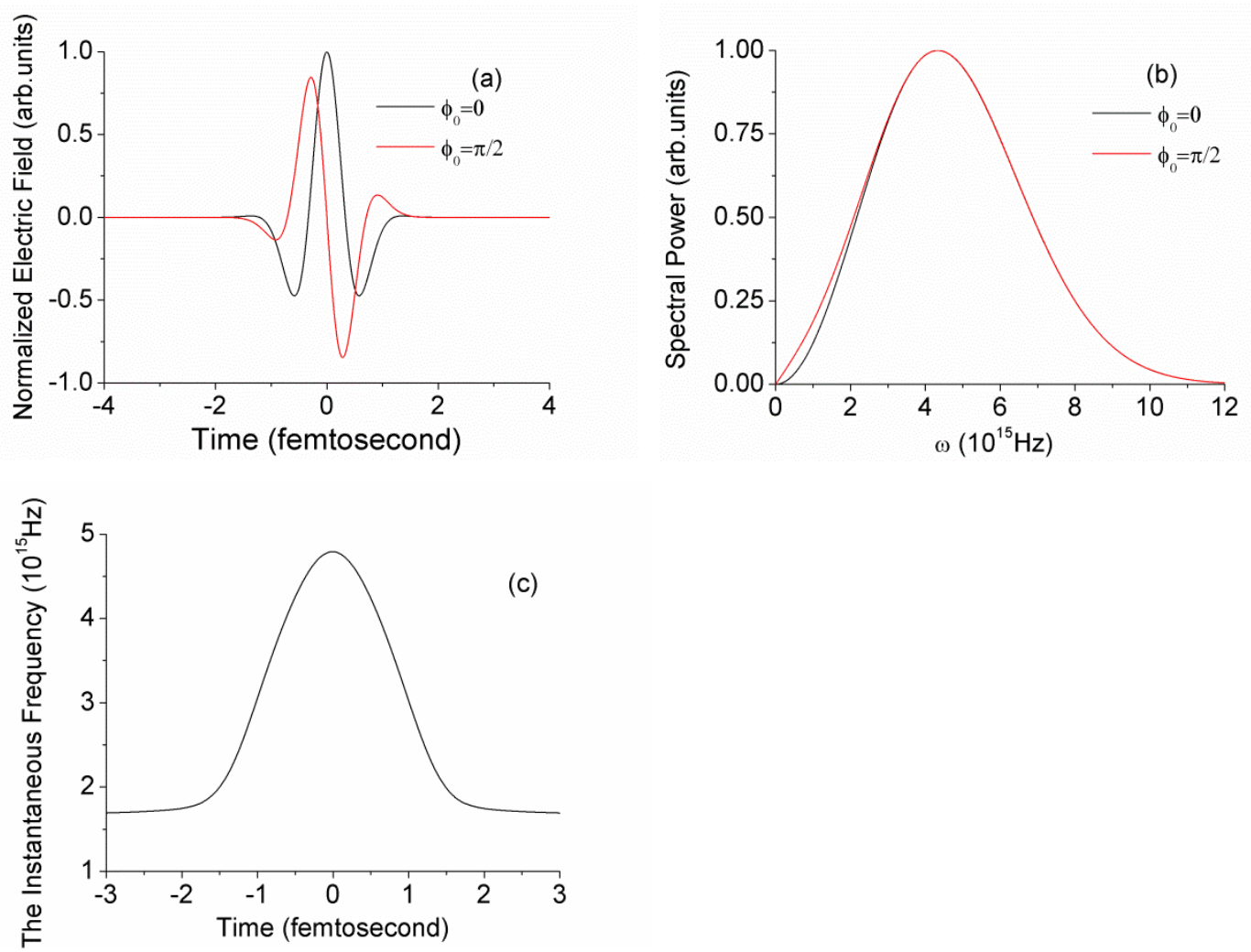
Fig.1(b) shows that center frequency of pulse remains unchanged when the CEP changes for pulse durations down to 0.28 cycle. The center frequency of pulse is $\omega=4.35 \times 10^{15} \mathrm{rad} / \mathrm{s}$, which is much larger than the carrier frequency. The instantaneous frequency changing over time is shown in Fig.1(c).

\section{Calculation method}

For the sub-cycle and single-cycle pulses, the SVE approximation is invalid, so the Maxwell's equations should be solved directly beyond the SVE approximation. The GVADE FDTD method can model spatial soliton propagation in fused silica and is an excellent algorithm. In this paper, the GVADE FDTD algorithm is used. Here is a brief introduction of the GVADE FDTD algorithm. The specific details can be found in Refs [13].

For the field components $\left\{E_{x}, H_{y}\right\}$ considered here, Maxwell's equations in one dimension are written as

$$
\frac{\partial E_{x}}{\partial z}=-\mu_{0} \frac{\partial H_{y}}{\partial t}, \frac{\partial H_{y}}{\partial z}=-\varepsilon_{0} \frac{\partial E_{x}}{\partial t}-J_{x}
$$

$\mathrm{z}$ is the direction of propagation. $E_{x}$ and $H_{y}$ are the electric and magnetic field components and $J_{x}$ is the polarization current, $\frac{\partial P_{x}}{\partial t}$. In materials with multiple-pole linear Lorentz polarization, instantaneous Kerr nonlear polarization, and Raman nonlinear polarization, the polarization current is $J_{x}=J_{\text {Lorentz }}+J_{\text {Kerr }}+J_{\text {Raman }}$. The linear Lorentz polarization contains a contribution from multiple resonances:

$$
J_{\text {Lorentz }}=\sum_{p=1}^{3} J_{\text {Lorentz }_{p}},
$$

where each $J_{\text {Lorentz }_{p}}$ corresponds to the polarization current due to a single pole of the Sellmeier expansion where the phasor polarization is given by

$$
\tilde{P}_{\text {Lorentz }_{p}}=\varepsilon_{0} \chi^{(1)} \tilde{E}=\varepsilon_{0} \frac{\beta_{p} \omega_{p}^{2}}{\omega_{p}^{2}-\omega^{2}} \tilde{E},
$$

where $\beta_{p}$ and $\omega_{p}$ are the pth resonance strength and frequency [8].

As a simple model of the electron response accounting for nonresonant in coherent nonlinear effects, the third order nonlinear polarization can be described by the Born-Oppenheimer approximation,

$$
P_{N L}(t)=\varepsilon_{0} \chi_{0}^{3} E \int_{-\infty}^{\infty} g\left(t-t^{\prime}\right)\left|E\left(t^{\prime}\right)\right|^{2} d t^{\prime}
$$

where $g(t)$ is the causal response function and $\chi_{0}^{3}$ is the third-order susceptibility coefficient. To model the kerr and the Raman polarizations, 


$$
g(t)=\alpha \delta(t)+(1-\alpha) g_{\text {Raman }}(t) \quad,
$$

where $\alpha$ represents the relative strengths of the Kerr and Raman polarizations, and

$$
g_{\text {Raman }}(t)=\left(\frac{\tau_{1}^{2}+\tau_{2}^{2}}{\tau_{1}^{2} \tau_{2}^{2}}\right) \exp \left(-t / \tau_{2}\right) \sin \left(t / \tau_{1}\right) U(t)
$$

where $g_{\text {Raman }}(t)$ is the Raman response function with parameters $\tau_{1}$ and $\tau_{2}$ chosen to fit the Raman-gain spectrum, and $U(t)$ is the Heaviside step function. From Eq.(14) and Eq.(15), the polarization and polarization current from the Kerr nonlinearity are

$$
\begin{aligned}
& P_{\text {Kerr }}(t)=\varepsilon_{0} \chi_{0}^{(3)} E \int_{-\infty}^{\infty} \alpha \delta\left(t-t^{\prime}\right)\left|E\left(t^{\prime}\right)\right|^{2} d t^{\prime}=\alpha \varepsilon_{0} \chi_{0}^{(3)}|E|^{2} E, \\
& J_{\text {Kerr }}(t)=\frac{\partial P_{\text {Kerr }}}{\partial t}=\frac{\partial}{\partial t} \alpha \varepsilon_{0} \chi_{0}^{(3)}|E|^{2} E .
\end{aligned}
$$

Writing Eq.(14) as a convolution, the polarization from Raman effect is

$$
P_{\text {Raman }}(t)=\varepsilon_{0} E\left[\chi_{\text {Raman }}^{(3)} *|E|^{2}\right],
$$

where

$$
\chi_{\text {Raman }}^{(3)}(t)=(1-\alpha) \chi_{\text {Raman }}^{(3)}(t) g_{\text {Raman }}(t) .
$$

According to Eqs.(12)-(20), the finite-difference expressions of linear Lorentz polarization, nonlinear Kerr polarization, nonlinear Raman polarization can be written as

$$
\begin{aligned}
& J_{\text {Lorentz }_{p}}^{n+1}=\alpha J_{\text {Lorentz }_{p}}^{n}-J_{\text {Lorentz }_{p}}^{n-1}+\gamma_{p} \frac{E^{n+1}-E^{n-1}}{2 \Delta t}, \\
& J_{\text {Kerr }}^{n+1 / 2}=\frac{\alpha \varepsilon_{0} \chi_{0}^{(3)}}{\Delta t}\left\{\left(\left|E^{n+1}\right|\right)^{2} E^{n+1}-\left(\left|E^{n}\right|\right)^{2} E^{n}\right\}, \\
& S^{n+1}=\left[\frac{2-\omega_{\text {Raman }}^{2}(\Delta t)^{2}}{\delta_{\text {Raman }} \Delta t+1}\right] S^{n}+\left[\frac{\delta_{\text {Raman }} \Delta t-1}{\delta_{\text {Raman }} \Delta t+1}\right] S^{n-1}+\left[\frac{(1-\alpha) \chi_{0}^{(3)} \omega_{\text {Raman }}^{2}(\Delta t)^{2}}{\delta_{\text {Raman }} \Delta t+1}\right]\left(\left|E^{n}\right|\right)^{2} \frac{n !}{r !(n-r) !}, \\
& J_{\text {Raman }}^{n+1 / 2}=\frac{\varepsilon_{0}}{\Delta t}\left(E^{n+1} S^{n+1}-E^{n} S^{n}\right),
\end{aligned}
$$

where the superscript indicates the time-step and $\alpha_{p}=2-\omega_{p}^{2}(\Delta t)^{2}, \gamma_{p}=\varepsilon_{0} \beta_{p} \omega_{p}^{2}(\Delta t)^{2} . S$ is a scalar auxiliary variable for the convolution,

$$
S(t) \equiv \chi_{\text {Raman }}^{(3)}(t) *|E(t)|^{2} .
$$

Form Eq.(11), one can get the expression

$$
\frac{H_{y}^{n+\frac{1}{2}}\left(i+\frac{1}{2}\right)-H_{y}^{n+\frac{1}{2}}\left(i-\frac{1}{2}\right)}{\Delta z}=-\left\{\frac{\varepsilon_{0}}{\Delta t}\left(E_{x}^{n+1}(i)-E_{x}^{n}(i)\right)+\sum_{p=1}^{3} J_{\text {Lorentz }}^{n+1 / 2}(i)+J_{\text {Kerr }}^{n+1 / 2}(i)+J_{\text {Raman }}^{n+1 / 2}(i)\right\},
$$

where the parameter $i$ indicates the space-step. Using the Newton's method, $E_{x}^{n+1}$ can be obtained by solving Eq.(26), where zeroes of objective functions are determined by iteration. Eq.(26) is solved by substituting Eq.(21), Eq.(22), and Eq.(24). We can define the objective function $X$ as 


$$
\begin{aligned}
X= & \frac{H_{y}^{n+\frac{1}{2}}\left(i+\frac{1}{2}\right)-H_{y}^{n+\frac{1}{2}}\left(i-\frac{1}{2}\right)}{\Delta z}+\frac{\varepsilon_{0}}{\Delta t}\left(E_{x}^{n+1}(i)-E_{x}^{n}(i)\right)+\frac{1}{2} \sum_{p=1}^{3}\left[\left(1+\alpha_{p}\right) J_{\text {Lorentz }_{p}}^{n}(i)-J_{\text {Lorentz }_{p}}^{n-1}(i)+\frac{\gamma_{p}}{2 \Delta t}\left(E_{x}^{n+1}(i)-E_{x}^{n-1}(i)\right)\right] \\
& +\frac{\alpha \varepsilon_{0} \chi_{0}^{(3)}}{\Delta t}\left\{\left(\left|E^{n+1}(i)\right|\right)^{2} E^{n+1}(i)-\left(\left|E^{n}(i)\right|\right)^{2} E^{n}(i)\right\}+\frac{\varepsilon_{0}}{\Delta t}\left(E^{n+1}(i) S^{n+1}(i)-E^{n}(i) S^{n}(i)\right)
\end{aligned}
$$

Next, we define $G_{x}^{g}$ to be the gth guesses for $E_{x}^{n+1}$. Newton's method updates the guesses until the objective function $\mathrm{X}$ are sufficiently close to zero. Each subsequent guess is made from the current guess by:

$$
G_{x}^{g+1}=G_{x}^{g}-\left.\left(J_{11}^{-1} X\right)\right|_{g},
$$

where

$$
J_{11}=\frac{\varepsilon_{0}}{\Delta t}+\frac{1}{4 \Delta t}\left(\gamma_{1}+\gamma_{2}+\gamma_{3}\right)+\frac{\varepsilon_{0}}{\Delta t}\left[3 \alpha \chi_{0}^{(3)} G_{x}^{2}+S^{n+1}\right] \text {, }
$$

and $\left.\right|_{g}$ indicates evaluation using the values from the gth guess.

\section{Simulation results}

The GVADE method is used to model temporal pulses in dispersive nonlinear materials. The initial expressions of pulses are shown in equation (7). The strengths and resonant frequencies of the linear dispersions from Sellmeier's equation are given by: $\beta_{1}=0.69617, \beta_{2}=0.40794, \beta_{3}=0.89748, \omega_{1}=2.7537 \times 10^{16} \mathrm{rad} / \mathrm{s}$, $\omega_{2}=1.6205 \times 10^{16} \mathrm{rad} / \mathrm{s}$, and $\omega_{3}=1.90342 \times 10^{14} \mathrm{rad} / \mathrm{s}$ [13]. The third-order electric susceptibility scalar is $\chi^{(3)}=1.89 \times 10^{-22}(\mathrm{~V} / \mathrm{m})^{-2}$. The relative strengths of the Kerr and Raman polarizations are given by the parameter $\alpha=0.7$.The Raman polarizations parameters are $\tau_{1}=12.2 f s$, and $\tau_{2}=32 f s$. The carrier frequency of pulse is $\omega_{c}=1.655 \times 10^{15} \mathrm{rad} / \mathrm{s}$. In Fig.2, the FWHM of pulse is $0.28 T_{0}$. Due to the self-induced blueshift of the center frequency of sub-cycle pulse, the center frequency of pulse is $\omega=4.35 \times 10^{15} \mathrm{rad} / \mathrm{s}$.The FDTD space resolution is $4 \mathrm{~nm}$ and the time resolution is $6.68 \times 10^{-3}$ femtosecond $(f s)$. The propagation of the 0.28 -cycle pulse is shown in Fig.2. The magnetic field was excited by a hard source at the far-left side of the grid $(z=0)$. The expression of magnetic field can be derived from equation (7) and the peak value of magnetic field is $H_{0}=4.77 \times 10^{7} \mathrm{~A} / \mathrm{m}$.
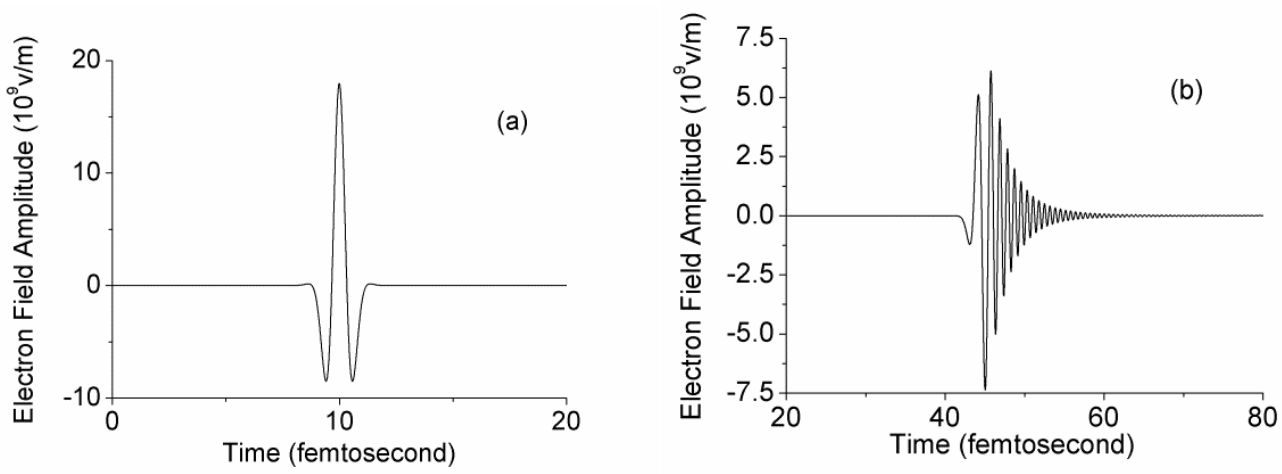
As is shown in Figure 2, the initial 0.28-cycle pulse evolves into a primary multi-cycle pulse and a single-cycle precursor pulse after the propagation time of 5.344 picoseconds. The single-cycle precursor pulse isn't completely separated from the primary multi-cycle pulse. From Figure 4(b), one can see that the central frequency of the single-cycle precursor pulse is much lower than that of the initial 0.28-cycle pulse. As the intrinsic characteristics of sub-cycle pulses, the instantaneous frequency of sub-cycle pulse is intrinsic chirp. The instantaneous frequency is symmetrical within the pulse and blueshifted with respect to the carrier-wave frequency $\omega_{c}$ throughout the entire pulse, and is the lowest both on the leading side and the trailing side of pulse. So the low frequency components produced by the carrier frequency modulation on the leading side of pulse is the fastest part during the propagation of the pulse. This is the reason of forming the single-cycle precursor pulse. The instantaneous frequency of sub-cycle pulse is highest at the center of the pulse $\left(>\omega_{c}\right)$ and the high frequency part of the pulse is the slowest part during the propagation. So the single-cycle precursor pulse gradually separates from the primary pulse during the propagation. The self-steepening effect is very clear for the sub-cycle pulse. The reason is that the maximum energy of the pulse is concentrated in the central part. The energy of the low frequency components produced by the carrier frequency modulation on the leading side of pulse is much less than that of the central part of pulse. The refractive index varies with the intensity of pulse. So the significant self-steepening effect is formed during the propagation of the sub-cycle pulse.

The self-steepening of the leading edge of a propagating pulse in a nonlinear medium can require a time-step that is smaller than what is needed for numerical stability. To verify the validity of the numerical simulation, the time-step in Fig.3(a) is reduced by 2:1 from its value in Fig.2, the grid resolution is kept. For the purpose of a final check, the grid resolution is $2 \mathrm{~nm}$ and the time step is $1.67 \times 10^{-3}$ femtosecond $(f s)$ in Fig.3(b). 
From Fig.3, one can see that the computation results are almost the same with Fig. 2. The single-cycle precursor pulse and the self-steepening effect are very clear. So the convergence of the numerical results relative to the FDTD time-step and grid resolution is checked.

The spectrums of the initial 0.28 -cycle pulse, the pulse in $480 \mu \mathrm{m}$ and the single-cycle precursor pulse are shown in Figure 4.
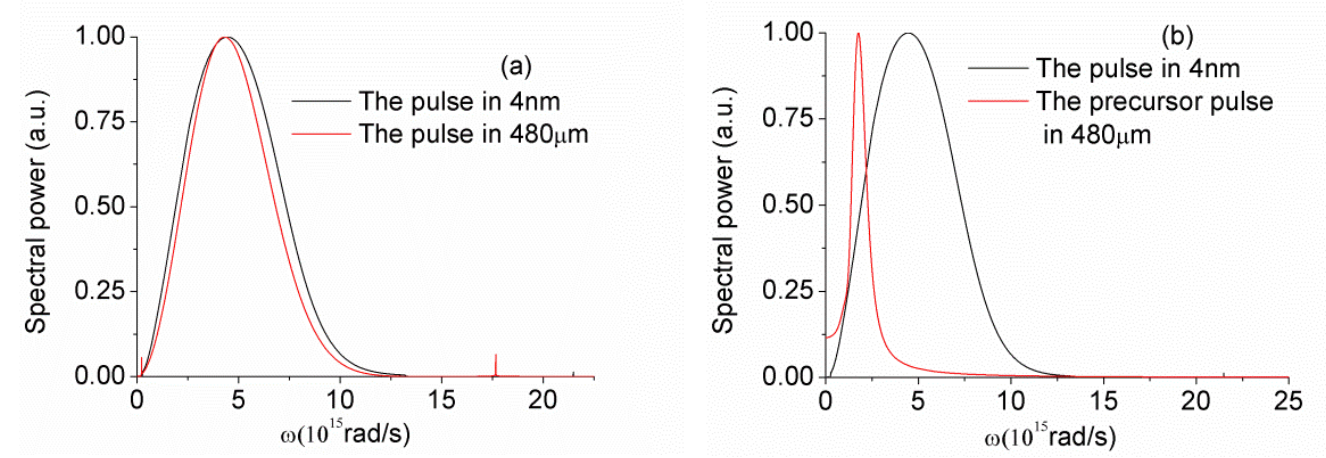

Fig. 4. (a) The spectrum of the pulse in $4 \mathrm{~nm}$ (black line) and the pulse in $480 \mu \mathrm{m}$ (red line).

(b) The spectrum of the pulse in $4 \mathrm{~nm}$ (black line) and the single-cycle precursor pulse in $480 \mu \mathrm{m}$ (red line).

As is shown in literature [22], the pulse becomes narrower, and the influence of the delayed Raman response gets smaller. The red-shift Raman peak can almost be ignored in Figure 4(a). Due to the carrier frequency modulation effect and the self-steepening effect, a portion of input energy is transferred into the low frequency precursor pulse. So the central frequency of the pulse is slightly red-shifted with respect to the initial pulse when the propagation distance of pulse is $480 \mu m$. 
Figure 5 shows that the initial 0.7 -cycle pulses evolve into a multi-cycle pulse after the propagation time of 5.344 picoseconds. From Fig.5(c), one can see that there is still a small precursor pulse on the leading side of pulse. But the amplitude of precursor pulse is reduced compared with that in Fig.5(c). It isn't easy to distinguish the precursor pulse from the primary pulse. With the increase of FWHM of the sub-cycle pulses, the intrinsic characteristics of pulses such as the intrinsic chirp and the self-induced blueshift are weakened, so the precursor pulse and the self-steepening effect of the 0.7 -cycle pulses are weakened compared with those of the 0.28 -cycle pulses.
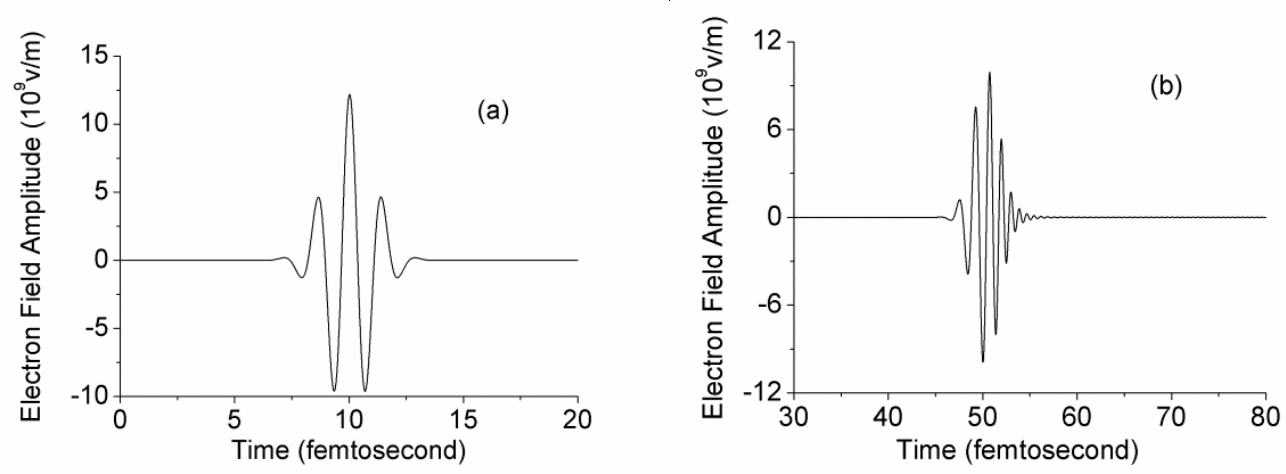

Fig. 5. The evolution of the 0.7-cycle pulse in the nonlinear dispersive media. (a), (b) The temporal profile of pulse. The propagation distance is $4 n m$ in (a) and $8 \mu \mathrm{m}$ in (b). (c) The spatial profile of pulse. The propagation time is 5.344 picoseconds in (c). Inset, the one-dimension spatial profile of the precursor pulse. 
Figure 6 shows that the initial 1.26-cycle pulses evolve into a multi-cycle pulse after the propagation time of 5.344 picoseconds. The precursor pulse can't be distinguished from the primary multi-cycle pulse. The reason is that the self-induced blue-shift of the central frequency of spectrum and the intrinsic chirp are weakened for the single-cycle pulse.

From Figure 2-6, one can see that the propagation of the analytical model of sub-cycle and single-cycle pulses contains rich content which can't be displayed in the carrier-envelope model. The reason is that the intrinsic properties of sub-cycle and single-cycle pulses are ignored in the carrier-envelope model. The initial chirp characteristics of the sub-cycle and single-cycle pulses are disappear during the propagation of the pulses. But due to the effect of the dispersion effect, the initial sub-cycle and single-cycle pulses change into positive chirp multi-cycle pulses. As previously mentioned, the single-cycle precursor pulse is formed by the carrier frequency modulation and the initial chirp characteristics of pulse. The evolutions of the initial 0.28-cycle pulses with different pulse peak intensities are shown in Fig.7.
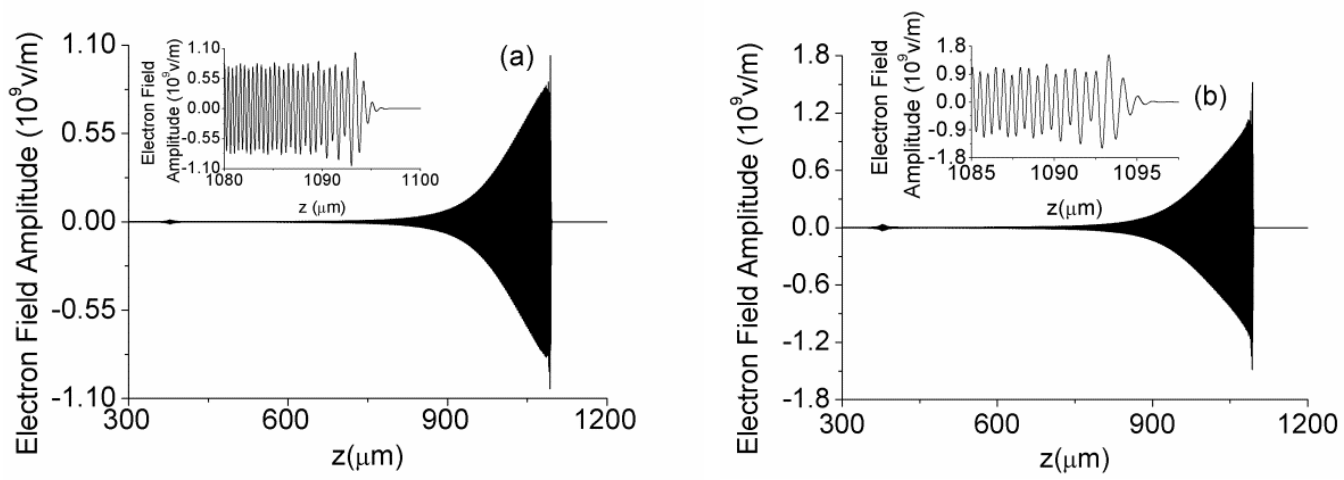

Fig. 7. The spatial evolution of the initial 0.28 -cycle pulse in the nonlinear dispersive media. The propagation time is 5.344 picoseconds. (a) The peak value of magnetic field is $H_{0}=6 \times 10^{7} \mathrm{~A} / \mathrm{m}$. (b) The peak value of magnetic field is $H_{0}=8 \times 10^{7} \mathrm{~A} / \mathrm{m}$. 
From Fig.7, one can see that the amplitudes of the single-cycle precursor pulses are larger when the peak intensities of the initial 0.28-cycle pulses are higher. The reason is that more energy of the initial pulse is transferred into the single-cycle precursor pulse due to the effect of the carrier frequency modulation.

\section{Conclusions}

In conclusion, we provide the analytical expressions of sub-cycle and single-cycle Gaussian pulses. In the analytical model, the DC part is removed and the Fourier spectrum $E(\omega)$ remains unchanged when the CEP changes for the pulse durations down to three-tenths cycle. Applying the analytical expressions of sub-cycle and single-cycle pulses, the propagation of the sub-cycle and single-cycle pulses in a nonlinear media is studied. The initial 0.28 -cycle pulse evolves into a primary multi-cycle pulse and a single-cycle precursor pulse after the propagation time of 5.344 picoseconds. The single-cycle precursor pulse is formed by the carrier frequency modulation on the leading side of pulse. The intrinsic characteristics of the sub-cycle pulse are found to have an important effect on the amplitude of the precursor pulse. The significant self-steepening effect is formed during the propagation of the sub-cycle pulse. With the increase of the FWHM of the sub-cycle pulses, the self-steepening effect and the amplitude of the precursor pulse are weakened. The reason is that the intrinsic characteristics of sub-cycle pulse such as the self-induced blue-shift of the central frequency of spectrum and the intrinsic chirp are weakened. With the increase of the peak intensities of the sub-cycle pulses, the amplitude of the single-cycle precursor pulses are larger, which is the result of the carrier frequency modulation and the initial chirp of the sub-cycle pulse.

\section{Aknowledgments}

We thank Prof. Allen Taflove, at Northwestern University, for helpful discussions. The work was supported by the Science Foundation of Guizhou Province, China (LKM(2013)19 and (2014)2090), the National Natural Science Foundation of China(NSF) (61475139), and the 973 Program of China (2013CB329501).

\section{References}

[1] M. Y. Shverdin, D. R. Walker, D. D. Yavuz, G. Y. Yin, and S. E. Harris, Phys. Rev. Lett. 94(2005) 033904.

[2] A. A. Voronin, J. M. Mikhailova, M. Gorjan, Zs Major, A. M. Zheltikov, Opt. Lett. 38(2013)4354.

[3] S. M. Feng , H. G. Winful, Phys. Rev. E 61(2000) 862.

[4] Z. Ulanowski, I. K. Ludlow, Opt. Lett. 25(2000)1792.

[5] P. Saari, Opt. Express 8(2001)590.

[6] Z. Wang, Q. Lin, Z. Wang, Phys. Rev. E 67(2003)016503.

[7] E. Goulielmakis, M. Schultze, M. Hofstetter, V. S. Yakovlev, J. Gagnon, M. Uiberacker, A. L. Aquila, E. M. Gullikson, D. T. Attwood, R. Kienberger, F. Krausz, U. Kleineberg, Science 320 (2008)1614.

[8] G. P. Agrawal, Nonlinear Fiber Optics, 5th ed. Academic, New York, 2012.

[9] S. Nakamura, N. Takasawa, Y.Koyamada, J. Lightwave Technol. 23(2005)855.

[10] C. V. Hile and W. L. Kath, J. Opt. Soc. Am. B 13(1996)1135. 
[11] V. P. Kalosha, J. Herrmann,Phys. Rev. A 62(2000)011804.

[12] P. M. Goorjian and A. Taflove, Opt. Lett. 17(1992) 180.

[13] J. H. Greene and A. Taflove, Opt. Exp. 14 (2006)8305.

[14] Y. Z. Xiao, D. N. Maywar, and G. P. Agrawal, Phys. Rev. A 87(2013) 063816.

[15] Y. Z. Xiao, D. N. Maywar, and G. P. Agrawal, J. Opt. Soc. Am. B. 29(2012) 2958.

[16] H. Leblond and D. Mihalache, Phys. Rep. 523(2013)61.

[17] D. J. Frantzeskakis, H. Leblond, and D. Mihalache, Rom. J. Phys. 59(2014)767.

[18] T. Brabec and F. Krausz, Phys. Rev. Lett. 78(1997) 3282.

[19] Q. Lin, J. Zheng, W. Becker, Phys. Rev. Lett.97(2006)253902.

[20] X. M. Cai, J. Zheng, Q. Lin, Phys. Rev. A 87(2013)043401.

[21] J. Zheng, E. Qiu, Y. Yang, and Q. Lin, Phys. Rev. A 85 (2012) 013417.

[22] E. Heyman, L. B. Felsen, J. Opt. Soc. Am. A 6(1989) 806. 